\title{
Echeneid-sirenian associations, with information on sharksucker diet
}

\author{
E. H. Williams JR*, A. A. Mignucci-Giannonitt, \\ L. Bunkley-Williams§, R. K. Bonde 1, C. Self-Sullivan**, \\ A. Preent广 and V. G. Cockcroft + t \\ * Department of Marine Sciences, University of Puerto Rico at Mayagüez, P. O. Box \\ 908, Lajas, Puerto Rico 00667-0908, †Caribbean Marine Mammal Laboratory, \\ Department of Science and Technology, Universidad Metropolitana, P. O. Box \\ 361715, San Juan, Puerto Rico 00936-1715, §Caribbean Aquatic Animal Health \\ Project, Department of Biology, University of Puerto Rico, P. O. Box 9012, Mayagüez, \\ Puerto Rico 00681-9012, ФSirenia Project, Florida Integrated Science Center, \\ U.S. Geological Survey, 412 NE 16th Avenue, Gainesville, Florida 32601-3701, \\ U.S.A., **Department of Wildlife and Fisheries Sciences, Texas A \& M University, \\ College Station, Texas 77843-2258, U.S.A., ††School of Tropical Environment Studies \\ and Geography, James Cook University, Townsville, Queensland 4811, Australia and \\ †Centre for Dolphin Studies, P. O. Box 1856, Plettenberg Bay 6600, South Africa
}

(Received 7 November 2002, Accepted 18 August 2003)

\begin{abstract}
Association of the sharksucker Echeneis naucrates and whitefin sharksucker E. neucratoides with the West Indian manatee Trichechus manatus and the dugong Dugong dugon is presented and discussed. Alimentary tract-content analysis and field observations suggest that coprophagy (feeding on host faecal material) may be the primary food source for echeneids associated with sirenian hosts.

(C) 2003 The Fisheries Society of the British Isles
\end{abstract}

Key words: commensalism; coprophagy; Dugong dugon; Echeneis; Trichechus manatus.

\section{INTRODUCTION}

The Echeneidae includes remoras, sharksuckers, whalesuckers and suckerfish, all known to associate commensally or mutualistically with pelagic and reef fishes, sharks, rays and cetaceans (O'Toole, 2002). Whalesuckers Remora australis (Bennett) are well-known associates of both whales and dolphins. Fertl \& Landry (1999) and Fertl et al. (2002) speculated that some of these records might be, in actuality, also of the sharksucker Echeneis naucrates L. The association of echeneids (sharksuckers) with sirenians, the dugong Dugong dugon (Müller) and three species of manatees Trichechus spp., has not been well-documented. Unidentified species of sharksuckers have occasionally been reported associated with West Indian manatees Trichechus manatus L. (Hartman,

\#Author to whom correspondence should be addressed. Tel.: +7877661717; fax: +787751 5840; email: mignucci@caribe.net 
1979), and dugongs (Husar, 1975; Brownell et al., 1981). A whitefin sharksucker Echeneis neucratoides Zouiev was collected from a West Indian manatee captured in Puerto Rico (Williams \& Bunkley-Williams, 1996; Mignucci-Giannoni et al., 1999). Feeding on host faecal material (coprophagy) was suggested in other echeneid species using fish hosts (Smith, 1950), but alimentary tract analysis in these species was not done or was inconclusive.

\section{MATERIALS AND METHODS}

Echeneids were collected during routine physical examinations incidental to West Indian manatee radio-tagging activities in Puerto Rico. Sharksuckers, often only loosely attached, were removed from manatees or from the net where manatees were captured. The fishes were placed in individual plastic bags, the bags covered with ice, and transported to a laboratory. Sharksuckers were identified to species by counting the paired lamellae on the disk following the key of Fischer (1978) and description in Williams \& Bunkley-Williams (1996). They were measured (standard, $L_{\mathrm{S}}$, and total length, $L_{\mathrm{T}}$ ), and their stomachs and intestinal tracts were examined for contents. One parasite species from these samples was reported by Bullard et al. (2000). Sub-samples of ingesta material were repeatedly rinsed, allowed to settle, and decanted until the solid matter in the sample could be examined using a dissection microscope. Two specimens of E. neucratoides and one specimen of E. naucrates were deposited in the U.S. National Museum (USNM) Ichthyology Collection, located at the Smithsonian Institution in Washington, D.C., U.S.A.

Counts and measurements were based on 25 sharksuckers and whitefin sharksuckers collected from manatees in Puerto Rico, 12 collected from manatees, measuredand-released in Florida, and 196 observations and size estimates from both localities. Sharksucker-manatee associations in Belize were documented at Gallow's Reef and the Drowned Cayes, within the Belize Barrier Reef lagoon system. Video images of manatees and sharksuckers were collected and analysed using a JVC MiniDV camera in an Ikelite underwater housing at 25 locations within the labyrinth of bogues, channels and seagrass beds that surround the Drowned Cayes and two locations on the nearby Gallow's Reef section of the Barrier Reef. Observations were also made incidental to manatee radiotagging activities in Southern Lagoon, a freshwater-estuarine system, located inland along the coast in central Belize.

Additionally, a total of hundreds of dugongs were observed in Australia, the Persian (Arabian) Gulf and Mozambique in south-eastern Africa. Video images of a herd of c. 120 dugongs were taken in the Persian Gulf off the western portion of the United Arab Emirates, and of 20 dugongs in the Bazaruto Bay in south-central Mozambique.

\section{RESULTS}

\section{WEST INDIAN MANATEES AS HOSTS}

For many years, sharksuckers Echeneis spp. on manatees have been observed in localities all around Puerto Rico (Fig. 1) (A.A. Mignucci-Giannoni pers. obs.). Twenty-five sharksuckers were collected from manatees captured in Puerto Rico. These were determined as E. naucrates $(n=17$, USNM 348299) and $E$. neucratoides ( $n=8$, USNM 348300). Both species were often found simultaneously on the same individual host specimen.

The occurrence of sharksuckers on manatees in Florida were generally rarer and the fishes were smaller than those found in Puerto Rico. In Puerto Rico, sharksuckers associated with c. $45 \%$ of the observed manatees, but on $<5 \%$ of 


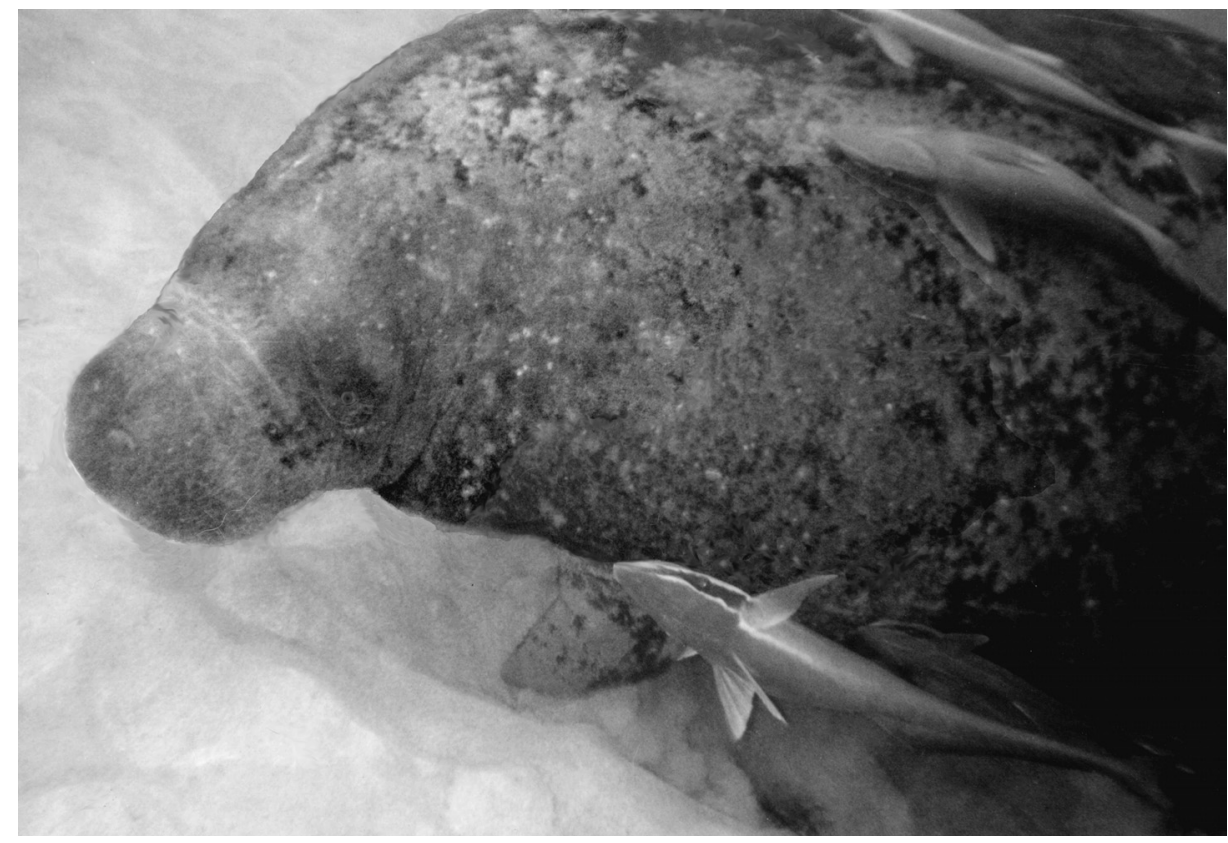

FIG. 1. Echeneis naucrates attached to a West Indian manatee Trichechus manatus off Aguadilla, Puerto Rico.

manatees observed in Florida. One to 21 sharksuckers occurred on each manatee in Puerto Rico, but only one to three in Florida. The $L_{\mathrm{S}}$ of the sharksuckers on manatees in Puerto Rico ranged from 51 to $73 \mathrm{~cm}$, while those from Florida were $25-35 \mathrm{~cm}$.

An unpublished museum record was found of a specimen of E. neucratoides collected from a manatee at Bocas de Laguna Guerrero, Bahía de Chetumal in the Mexican Caribbean (Zoology Musuem, El Colegio de la Frontera Sur, ECO-CH-2785, 24 May 1995).

In Belize, 103 images of manatees were 'captured' on 73 underwater video clips from 1999 to 2002 in the Drowned Cayes area, a marine habitat (C. SelfSullivan, unpubl. data). Sharksuckers were observed in association with manatees in 49 of the clips. Sharksuckers were visually identified as Echeneis spp. These results are probably an under estimate of actual associations due to the quality of video samples, which varied due to visibility, distance from observer to target animal, and number of angles captured on tape. The number of sharksuckers associated with each manatee ranged from one to 16 . No sharksuckers were observed in association with a manatee in 54 images, whereas one or more sharksuckers were observed in association with a manatee in 49 images ( $48 \%$ of the total number of captured images). When sharksuckers were visible, the number of sharksuckers associated with one manatee for most cases ranged from one to five. In three videos, however, from eight to 16 sharksuckers were observed associated with a manatee. In contrast, of 24 individual manatees captured for radio-tagging a total of 61 times over a 5 year period from a 
freshwater-estuarine system in Central Belize, only one net set produced small 25-30 cm E. neucratoides associated with the target animal.

\section{DUGONGS AS HOSTS}

Husar (1975) reported an unidentified species of sharksucker on a dugong off Green Island, Australia. Brownell et al. (1981) noted unidentified sharksuckers on dugongs in Palau. Sharksuckers and possibly other species are common on dugongs in Australia, but rather rare in Mozambique and the Persian Gulf. Only four small $(c .20-30 \mathrm{~cm})$ sharksuckers, one-each on four of six or seven dugongs, were observed on one occasion in Bazaruto Bay, Mozambique (V.G. Cockcroft, pers. obs.). Quite a few dugongs in a herd of 120 had sharksuckers associated with them in the western portion of the United Arab Emirates in the Persian Gulf. In Australia, sharksuckers on dugongs ranged in size from c. 30 (rarely smaller) to $100 \mathrm{~cm} L_{\mathrm{T}}$. Most were E. naucrates, although other sharksucker species could be present. Up to four sharksuckers, often of different sizes, occurred on a single dugong. One large sharksucker $\left(>80 \mathrm{~cm} L_{\mathrm{T}}\right)$ was seen attached to a recently orphaned dugong calf that was $1.5 \mathrm{~m}$ in body length. Sharksuckers attached to all areas of the trunk of dugongs, caudal from the head. They were often tenacious in their attachment. When dugongs were pursued for capture and tagging, large sharksuckers (up to $100 \mathrm{~cm} L_{\mathrm{T}}$ ) usually released from the dugong when it performed somersaults and sharp turns to avoid capture; however, smaller sharksuckers (up to $50 \mathrm{~cm} L_{\mathrm{T}}$ ) often remained with the dugong when it was caught and during the tagging process unless the host was removed from the water.

\section{DISCUSSION}

\section{SHARKSUCKER ASSOCIATION WITH SIRENIANS}

The association of echeneids to manatees was first recorded in the Caribbean in accounts by early explorers (Fernández de Oviedo y Váldez, 1526; Durand, 1983) and recently in Florida (Hartman, 1979). Sharksuckers associated with manatees were often assumed to be the E. naucrates, but this species is difficult to distinguish from the E. neucratoides in the field. Despite numerous previous studies of the manatee, the identity of its sharksucker associates was not previously confirmed. Fertl \& Landry (1999) and Fertl et al. (2002) found a similar situation with the sharksuckers of dolphins. The species had been assumed to be the whalesucker, but through more careful examination, they found cases of the E. naucrates on bottlenose dolphins Tursiops truncatus Montagu and spinner dolphins Stenella longirostris (Gray, 1828).

Manatees with sharksuckers attached were found along all coasts of Puerto Rico where manatees occur only in salt water. In Florida, this association occurred on both coasts and in marine, brackish, and freshwater habitats. Robins et al. (1991) consider that echeneids are not capable of invading fresh water, however, E. naucrates is well documented to enter fresh water in Lake Nicaragua (Thorson, 1976). Possibly the Florida and Nicaragua records are sufficient to establish E. naucrates' ability to invade freshwater habitats. 
Like many shark, dolphin and whale species, dugongs and manatees may be a particularly attractive 'habitat' for sharksuckers. Adult dugongs and manatees are large (up to $3 \mathrm{~m}$ long and $400 \mathrm{~kg}$ ) and appear to be relatively safe from predation by most non-human predators. It seems that sharksucker association in dugongs may be related to the host's group size. Dugongs often occur in large herds. It may not be energetically efficient for a sharksucker to chase after a single, or just a few dugongs, and associating with larger groups of dugongs may make it easier to change hosts, as has been observed in dolphins. Additionally, it is speculated that dugongs and manatees may be desirable hosts as their seagrass-feeding behaviour may provide small prey items for their attached sharksuckers. Dugongs typically create furrows that are $c .15 \mathrm{~cm}$ wide and 5-10 cm deep and up to $10 \mathrm{~m}$ long (Preen, 1993), while manatees uproot material from large elliptical patches averaging $27 \mathrm{~m}^{2}$ in area (Lefebvre et al., 2000). These processes are destructive and they flush fishes and crustaceans and expose worms, molluscs and other invertebrates. Feeding dugongs often attract other fishes and cormorants that also exploit this potential source of food.

Some species of sharksuckers are known to have flexible host-specificity and dugongs and manatees often associate with other host species, potentially allowing host-switching by sharksuckers. Large (3-4 m) sharks are occasionally seen within $100 \mathrm{~m}$ of dugong herds and sea turtles and large rays associate closely with dugongs. Dolphins and sea turtles share some of their habitat with manatees in Florida and Puerto Rico and may also provide transport as they are often found in close proximity. It is an intriguing possibility that facultatively coprophagic sharksuckers may be able to vary the nature of their diet by switching between herbivorous and carnivorous hosts.

The sharksuckers associated with both manatees and dugongs appear to be generally larger and more common in the tropics (Puerto Rico and Australia, respectively) than in the subtropical and temperate regions (Florida and southeast Africa, respectively). At present, there is little information to suggest a cause for this trend.

\section{FOOD HABITS OF SHARKSUCKERS}

The stomach and intestinal contents of 17 specimens of E. naucrates and eight specimens of E. neucratoides found associated with manatees in Puerto Rico were analysed. Almost all of the ingesta of the sharksuckers examined consisted of host faeces. The consistency and texture of the material in the stomach was characteristic of the very digested ingesta typically passed through the host and not course and abraded as might be expected of material culled directly from the rooted plants (Burn, 1986; Marsh et al., 1999). Numerous instances of sharksuckers associated with wild manatees in Florida feeding directly on manatee faeces just after host defecation were also observed.

Smith (1950) suggested that sharksuckers consumed the faeces of their hosts based on earlier observations by others of their feeding behaviour. Strasburg (1959) belittled Smith's (1950) opinion and established the modern opinion of opportunistic feeding on host-scraps, plankton and parasites. More information on the amounts (in terms of total diet) of parasite-picking was added later by Cressey \& Lachner (1970). The present analysis of stomach and intestinal 
contents of sharksuckers associated with manatees in Puerto Rico, as well as direct observation of sharksuckers feeding on manatee faeces just after defecation in Florida, suggest that Smith (1950) was correct.

The debate between Smith (1950) and Strasburg (1959) over faecal $v$. scrap and plankton feeding strategies in sharksuckers cannot be resolved by the analysis of stomach and intestinal contents of those sharksuckers associated with a piscivorous host. Generally, those scraps from the host during feeding, basically ingested planktonic fishes, and the hosts' faecal contents all contain similar things that are indistinguishable in the stomach and faecal contents of those sharksuckers. The contents of sharksuckers associated with manatees and dugongs can be analysed conclusively, however, and can be used to resolve the general question. Sirenians eat submerged aquatic vegetation, not fishes or planktonic organisms. The stomach and intestinal contents of the sharksuckers examined contained a large volume of pre-digested plant material, of a consistency that would suggest that it had already passed through the manatee's efficient digestive tract (only three small fish vertebrae occurred in one of 25 sharksuckers). Sharksuckers are known to have only fish remains in their stomach contents when found associated with piscivorous hosts. It is unlikely that they become almost exclusively herbivorous just because they associate with a plant-eating host. The most logical conclusion is that sharksuckers feed almost exclusively on the faeces of their hosts. Sharksuckers on piscivorous hosts have fish remains in their stomachs and intestines, while those on herbivorous hosts have plant material. The present evidence agrees with Smith's (1950) suggestion that a large part of the diet of sharksuckers was faecal material. Furthermore, the diet of sharksuckers associated with the manatees examined in the present study is almost exclusively the faecal materials voided by their hosts. This may also be true of sharksuckers associated with other hosts.

Supportive of this theory is the rather elaborate intestinal tract of sharksuckers that is not typical of a piscivorous carnivore that feeds only on fish scraps, but is more suited to an animal consuming a variety of materials such as faecal material from different host species with widely varying diets. The optional digestion hypothesis predicts herbivorous fishes should have a longer digestive tract than carnivorous fishes (Helfman et al., 1997). Kramer \& Bryant (1995) found that at a given size, intestinal lengths of herbivores are longer than those of omnivores, which in turn are longer than those of carnivores. Benavides et al. (1994) demonstrated that an increase in relative gut length is associated with an increase in the capacity to digest plant matter. Additional studies should be conducted to rule out possible seasonal biases associated with plant availability and host habitat influences that could ultimately affect the foraging material available to sharksuckers. It would be interesting, for example, to document how sharksuckers cope with changing diets between a sirenian and a shark or dolphin host, and if individual sharksuckers can make the change in mid-life once the fish's gut flora is established and suited to a particular diet. Furthermore, additional studies, perhaps with captive animals of both sirenians and sharksuckers, may shed light on the implications of a derived vegetarian diet for sharksuckers given the low protein content of digested plant material that could ultimately affect for example growth and fecundity. 
We gratefully acknowledge the assistance of Caribbean Stranding Network participants and volunteers, particularly N.M. Lee, M. Thomas, K.V. Hall, P. Acevedo, N. Ramírez-Sacarello and N. Jiménez-Marrero. Manatee captures in Puerto Rico were made under permits from the U.S. Fish and Wildlife Service (PRT-684532, 1991-1994 PRT 791721, 1996-1999), and Puerto Rico's Department of Natural and Environmental Resources. Data from the Drowned Cayes area of Belize were collected under annual permits granted by the Conservation Division of the Belizean Forestry Department. J.A. Powell graciously provided data from manatee observations during capture and handling of manatees in Belize's Southern Lagoon. Support was provided in part to the different projects by grants from the U.S. Fish and Wildlife Service's Caribbean Field Office, U.S. Naval Station at Roosevelt Roads, Oceanic Society, American Museum of Natural History's Lerner-Gray Marine Research Fund, Earthwatch Institute, New England Aquarium's Conservation Action Fund, the National Science Foundation's Model Institutions for Excellence (MIE) Project and Graduate Fellowship Program, and a grant from the Commonwealth of Puerto Rico's Legislature. We thank D. Fertl, A. Ballard and J.P. Reid for reviewing the manuscript.

\section{References}

Benavides, A. A., Cancino, J. M. \& Ojeda, F. P. (1994). Ontogenetic changes in gut dimension and macroalgae digestibility in the marine herbivorous fish, Aplodactylus punctatus. Functional Ecology 8, 46-51.

Brownell, R. L., Anderson, P. K., Owen, R. P. \& Ralls, K. (1981). The status of dugongs at Palau, an isolated island group. In The Dugong. Proceedings of a Seminar/ Workshop Held at James Cook University of North Queensland (Marsh, H., ed.), pp. 19-42, Townsville: James Cook University.

Bullard, S. A., Benz, G. W., Overstreet, R. M., Williams, E. H. Jr \& Hemdal, J. (2000). Six new hosts records and an updated list of wild hosts for Neobenedia mellani (Maccallum, 1927) (Monogenea: Capsalidae). Comparative Parasitology 67, 190-196.

Burn, D. M. (1986). The digestive strategy and efficiency of the West Indian manatee, Trichechus manatus. Comparative Biochemistry and Physiology 85A, 139-142.

Cressey, R. F. \& Lachner, E. A. (1970). The parasitic copepod diet and life history of diskfishes (Echeneidae). Copeia 1970, 310-318.

Durand, J. (1983). Ocaso de Sirenias: Esplendor de Manaties. Mexico: Fondo de Cultura Economica.

Fernández de Oviedo y Váldez, G. (1526). Dela Natural Hystoria delas Indias. Toledo: Ramón de Petras.

Fertl, D. \& Landry, A. M. (1999). Sharksucker (Echeneis naucrates) on a bottlenose dolphin (Tursiops truncatus), and a review of other cetacean-remora associations. Marine Mammal Science 15, 859-863.

Fertl, D., Landry, A. M. \& Barros, N. B. (2002). Sharksucker (Echeneis naucrates) on a bottlenose dolphin (Tursiops truncatus) from Sarasota Bay, Florida, with comments on remora-cetacean associations in the Gulf of Mexico. Gulf of Mexico Science 20, 151-152.

Fischer, W. (1978). FAO Species Identification Sheets for Fishery Purposes. Western Central Atlantic (Fishing Area 31), Vols 1-7. Rome: Food and Agriculture Organization of the United Nations.

Hartman, D. S. (1979). Ecology and Behavior of the Manatee (Trichechus manatus) in Florida. Pittsburgh, PA: American Society of Mammalogists.

Helfman, G. S., Collette, B. S. \& Facey, D. E. (1997). The Diversity of Fishes. Malden, MA: Blackwell Science.

Husar, S. (1975). A review of the literature of the dugong (Dugong dugon). Wildlife Research Reports (U.S. Fish and Wildlife Service) 4, 1-30. 
Kramer, D. L. \& Bryant, M. J. (1995). Intestine length in the fishes of a tropical stream: 2. Relationship to diet the long and short of a convoluted issue. Environmental Biology of Fishes 42, 129-141.

Lefebvre, L. W., Reid, J. P., Kenworthy, W. J. \& Powell, J. A. (2000). Characterizing manatee habitat use and seagrass grazing in Florida and Puerto Rico: implications for conservation and management. Pacific Conservation Biology 5, 289-298.

Marsh, H., Beck, C. A. \& Vargo, T. (1999). A comparison of the capabilities of dugongs and West Indian manatees to masticate seagrasses. Marine Mammal Science 15, 250-255.

Mignucci-Giannoni, A. A., Beck, C. A., Montoya-Ospina, R. A. \& Williams, E. H. Jr (1999). Parasites and commensals of the West Indian manatee from Puerto Rico. Journal of the Helminthological Society of Washington 66, 67-69.

O'Toole, B. (2002). Phylogeny of the species of the superfamily Echeneoidea (Perciformes: Carangoidei: Echeneidae, Rachycentridae, and Coryphaenidae), with an interpretation of echeneid hitchhiking behaviour. Canadian Journal of Zoology 80, 596-623.

Preen, A. R. (1993). Interactions between dugongs and seagrasses in a subtropical environment. PhD Dissertation, James Cook University of North Queensland.

Robins, C. R., Bailey, R. M., Bond, C. E., Brooker, J. R., Lachner, E. A., Lea, R. N. \& Scott, W. B. (1991). Common and Scientific Names of Fishes from the United States and Canada, 5th edn. American Fisheries Society, Special Publication 20.

Smith, J. L. B. (1950). The Sea Fishes of Southern Africa. Capetown: Central News Agency.

Strasburg, D. W. (1959). Notes on the diet and correlating structures of some central Pacific echeneid fishes. Copeia 1959, 244-248.

Thorson, T. B. (1976). Investigations of the Ichthyofauna of Nicaraguan Lakes. Lincoln, NE: School of Life Sciences, University of Nebraska.

Williams, E. H. Jr \& Bunkley-Williams, L. (1996). Parasites of Offshore, Big Game Sport Fishes of Puerto Rico and the Western North Atlantic. Mayagüez: Puerto Rico Department of Natural and Environmental Resources and Department of Biology, University of Puerto Rico. 